\title{
https://doi.org/10.46813/2021-134-144 \\ CONTROL OF WAVE INTERACTION PROCESSES IN PLASMA
}

\author{
V.O. Buts ${ }^{2,3}$, I.K. Kovalchuk ${ }^{2}$, O.P. Tolstoluzhsky ${ }^{2}$, A.G. Zagorodny ${ }^{1}$ \\ ${ }^{1}$ Bogolyubov Institute for Theoretical Physics, National Academy of Sciences of Ukraine, \\ Kyiv, Ukraine; \\ ${ }^{2}$ National Science Center “Kharkov Institute of Physics and Technology”, Kharkiv, Ukraine; \\ ${ }^{3}$ Institute of Radio Astronomy of NAS of Ukraine, Kharkiv, Ukraine \\ E-mail:vbuts@kipt.kharkov.ua
}

The results of processes investigation of nonlinear interaction of wave in plasma were expounded. First of all the mechanisms of the processes control of three frequencies wave interaction were considered. It was shown that using of the whirligig principle allows to point out to conditions of existence of decay processes and to conditions of suppression of decay processes. It was discovered that separated waves with circle polarization do not decay in plasma.

PACS: 52.35.Mw

\section{INTRODUCTION}

Processes in plasma are separated into two fundamental directions: processes of interaction wave-particle type and one of wave-wave type. In the last case processes of wave interaction can be divided on parametric processes and nonlinear ones. Processes of three wave interaction in plasma should be highlighted from nonlinear ones. These processes are mostly studied and most often appear in experiment. Such feature of these interactions is connected that they are conditioned by the quadratic nonlinearity. The nonlinearities of more higher order do not observe in experiment (they are small and proportional to third and more higher power of small parameters). Essential meaning among three wave interaction in plasma have decays. They allow to transform energy of high frequency waves into energy of low frequency ones that effectively heat plasma. This is positive function of decays. On other hand they can prevent to propagation and accumulation wave energy in the plasma waveguides and cavities. Such to effectively to control the characteristics of interacting waves it is necessary to know conditions that will support decays and that will support these processes. In this work the answers for some of these questions will be presented. The principle of whirligig was putted into base of finding of effective mechanism for control of wave interaction processes in plasma $[1,2]$.

\section{STABILIZATION OF THREE WAVE DECAY PROCESS IN PLASMA}

As example of using of whirligig principle let us consider stabilization of well studied (see, for example, $[3,4])$ three wave process in plasma. The set of equations that describes investigating processes will look like this

$$
\dot{A}_{0}=\sigma_{0} A_{1} A_{2} ; \dot{A}_{1}=\sigma_{1} A_{0} A_{2}^{*} ; \dot{A}_{2}=\sigma_{2} A_{0} A_{1}^{*}+\mu A_{3} ; \dot{A}_{3}=-\mu A_{2} .
$$

Here $A_{0}$ - amplitude of wave that decays, with maxima frequency $\omega_{0} ; A_{1}$ - amplitude of high frequency wave, on that take place decay. The frequency of this wave equals $\omega_{1} ; A_{2}$-amplitude of low frequency wave that take part in the process of three wave deacay. The frequency of this wave equals $\Omega$. There is synchronism between waves $\left(\omega_{0}=\omega_{1}+\Omega\right)$. $A_{3}$ - amplitude of fourth wave that as we suppose must suppress decay process. This wave have frequency that equals to one of low frequency wave and is synchronous with them and interaction with them with coefficient that equals $\mu$.

We will to study the initial study of decay. In this case amplitude of decaying wave can be considered as constant $\left(A_{0}=\right.$ const $)$. Then equation for amplitude $A_{2}$ is obtained from set of equation (1):

$$
\ddot{A}_{2}+\left[\mu^{2}-\sigma_{1} \sigma_{2}\left|A_{0}\right|^{2}\right] A_{2}=0 .
$$

From equation (2) it is seen that decay process will be suppressed if following condition will be satisfied:

$$
\mu^{2}>\sigma_{1} \sigma_{2}\left|A_{0}\right|^{2} \text {. }
$$

Taking into account, that first addend in square brackets is conditioned by the linear process and second is conditioned by nonlinear process then inequality is satisfied easily.

Inequality (3) was obtained by using additional stabilizing perturbance $\left(A_{3}\right)$. The whirligig principle may be us full to find out conditions of conditions decay existence and to find out conditions of suppression. As example of such analysis we will consider processes of nonlinear three wave interaction in the Magnetoactive plasma.

\subsection{FORMULATION OF PROBLEM. MAIN EQUATION}

Common initial equations are Maxwell ones for field, continuity equation for plasma density and equation of motion for plasma particles:

$$
\begin{aligned}
& \operatorname{rot} \vec{H}=\frac{1}{c} \frac{\partial \vec{E}}{\partial t}+\frac{4 \pi}{c} \vec{j}, \quad \operatorname{rot} \vec{E}=-\frac{1}{c} \frac{\partial \vec{H}}{\partial t}, \quad \vec{j}=e n \vec{v}, \\
& \frac{\partial n}{\partial t}+\nabla(n \vec{v})=0, \quad \frac{\partial \vec{v}}{\partial t}+(\vec{v} \nabla) \vec{v}=\frac{e}{m} \vec{E}+\frac{e}{m c}[\vec{v} \vec{H}] .
\end{aligned}
$$

To avoid of unwieldy formulae, physical content of which is difficultly to understand, we in this section limit our consideration by one dimensional case, i.e. the derivatives that do not equals zero are temporal one and one of the space derivative: $\partial / \partial z \neq 0$ and $\partial / \partial t \neq 0$. Components of field we select in the form: $\left(E_{x}, H_{y}\right) ;\left(E_{y}, H_{x}\right)$ - high frequency $(H F)$ field, 
$E_{z}$ - longitudinal low frequency $(L F)$ field,

and also $H_{0}=H_{z}=$ const - constant external magnetic field in which plasma is placed

In the magnetoactive plasma natural modes are waves with circular polarization. Keep in mind this we introduce new dependent variables:

$$
E_{ \pm}=E_{x} \pm i E_{y} ; \quad H_{ \pm}=H_{x} \pm i H_{y} ; \quad v_{ \pm}=v_{x} \pm i v_{y} .
$$

Set of equations (4) for this new dependent variables is transformed into next one:

$$
\begin{aligned}
& \frac{\partial E_{ \pm}}{\partial z}=-\frac{1}{c} \frac{\partial}{\partial t}\left(H_{y} \mp i H_{x}\right)= \pm i \frac{1}{c} \frac{\partial}{\partial t} H_{ \pm}, \\
& \frac{\partial H_{ \pm}}{\partial z}=\mp \frac{i}{c} \frac{\partial}{\partial t} E_{ \pm} \mp i \frac{4 \pi e n}{c} v_{ \pm}, \\
& \frac{\partial}{\partial t}\left(v_{ \pm}\right)+v_{z} \frac{\partial}{\partial z}\left(v_{ \pm}\right)=\frac{e}{m}\left(E_{ \pm}\right) \mp i \omega_{H}\left(v_{ \pm}\right) \pm i \frac{e}{m c} v_{z}\left(H_{ \pm}\right) .
\end{aligned}
$$

where $\omega_{H}=e H_{0} / m c-$ electron cyclotron frequency.

If dependent variables from coordinate $z$ is as $\left(\sim e^{-i k z}\right)$, equations (7) are ones for two nonlinear, connected oscillators for two HF waves:

$$
\begin{aligned}
& \frac{\partial^{2} E_{ \pm}}{\partial z^{2}}-\frac{1}{c^{2}} \frac{\partial^{2} E_{ \pm}}{\partial t^{2}}-\frac{4 \pi e^{2} n_{0}}{m c^{2}} E_{ \pm}=(\mp i) \frac{4 \pi e n_{0}}{c^{2}} \omega_{H} v_{ \pm} \\
& +\frac{4 \pi e n_{1}}{c^{2}}\left[\frac{e}{m} E_{ \pm} \mp i \omega_{H} v_{ \pm}\right]-\frac{4 \pi e n_{0}}{c^{2}}\left[v_{z}\left(\frac{\partial v_{ \pm}}{\partial z} \mp i H_{ \pm}\right)\right] \\
& \frac{\partial}{\partial t}\left(v_{ \pm}\right)+v_{z} \frac{\partial}{\partial z}\left(v_{ \pm}\right)=\frac{e}{m}\left(E_{ \pm}\right) \mp i \omega_{H}\left(v_{ \pm}\right) \pm i \frac{e}{m c} v_{z}\left(H_{ \pm}\right) .
\end{aligned}
$$

Here $\omega_{p}=\sqrt{4 \pi e^{2} n_{0} / m}$ is plasma frequency which is frequency of excited LF-wave.

Dispersion curves for electromagnetic and plasma waves is presented on Figs. 1, 2. Arrows point out possible resonantly interacting triplets of wave.

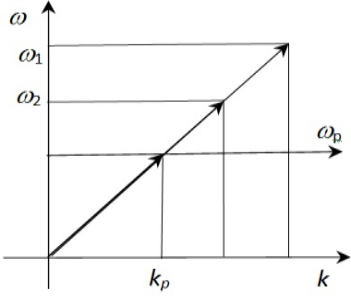

Fig. 1. Dispersion

diagram of wave that propagate in one direction

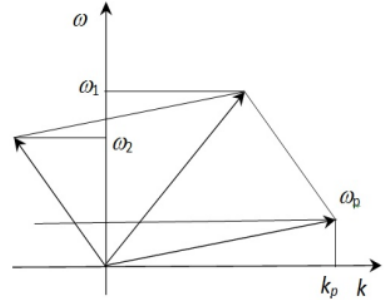

Fig. 2. Dispersion diagram of wave that propagate different directions
Latter we will neglect by dependence from space coordinate (resonator case). In this case all dependence versus coordinate can be rewrited as $\partial G / \partial z=-i k G$ for high frequence variables, and $\partial G / \partial z=-i \kappa G$ for low frequency ones. In this case set of equations for low frequency variables has the form:

$$
\begin{gathered}
\frac{\partial n_{1}}{\partial t}=i \kappa v_{z}\left(n_{0}+\tilde{n}\right) \\
\frac{\partial v_{z}}{\partial t}=-\left(\frac{4 \pi e}{i \kappa}\right) \frac{e}{m} \tilde{n}+i \kappa v_{z}^{2}+\left(\frac{e}{2 m c}\right)\left(\frac{k c}{\omega}\right)\left[v_{-} E_{+}+v_{+} E_{-}\right]
\end{gathered}
$$

In the nonlinear addend of set (10), (11) it may substitute expressions that obtained from linear equations.

Solution of equations (8), (9) we will find in the form:

$$
E_{j}=\left[A_{j}(t) \exp \left(i \varphi_{j}\right)+k . c .\right], j=\{1,2,3\} .
$$

Here $\varphi_{j}=\left(\omega_{j} t-k_{j} z\right) ; \omega_{j}$ and $k_{j}$ frequency and wave vector of interacting wave. These wave characteristic satisfy to linear dispersion equation. Amplitudes $A_{j}(t)$ slowly vary as a result of nonlinear wave interaction. Frequencies and wave vectors of interacting waves must satisfy to famous synchronism conditions:

$$
\omega_{1}=\omega_{2}+\omega_{3}, \quad \vec{k}_{1}=\vec{k}_{2}+\vec{k}_{3} .
$$

\subsection{DECAY OF TYPE $t \rightarrow t+l$ TRANSVERCE WAVE WITHOUT EXTERNAL MAGNETIC FIELD $\omega_{H}=0$}

First of all we will consider most simple and well studied decay of transverse wave into transverse one and plasma one when external magnetic field is absent $\omega_{H}=0$. Dispersion diagrams waves are presented on Figs. 1,2. This is decay of $t \rightarrow t+l$ type. In this case it may be limited by studying of dynamics of linear polarization wave with components $E_{x}, H_{y}\left(E_{y}=H_{x}=0\right)$. Then $E_{ \pm}=E_{x} ; v_{ \pm}=v_{x} ; H_{ \pm}=i H_{y}$.

For analysis of slow nonlinear dynamics of amplitudes of interacting waves from equations $(8-11)$ by usual method [3 - 5] the following set of shorted equations to defining of these amplitudes is obtained:

$$
\begin{aligned}
\frac{\partial A_{1}}{\partial t}=-\frac{\omega_{p} \kappa}{2 \omega_{2}} A_{2} a_{3} & \quad \frac{\partial A_{2}}{\partial t}=\frac{\omega_{p} \kappa}{2 \omega_{1}} A_{1} a_{3}^{*} \\
\frac{\partial a_{3}}{\partial t}= & \left(\frac{e}{m}\right)^{2} \frac{\left(k_{1}+k_{2}\right)}{2 \omega_{1} \omega_{2}} A_{1} A_{2}^{*} .
\end{aligned}
$$

In this set for slow function of longitudinal velocity the new designation was introduced:

$$
A_{3}=a_{3} \text {. }
$$

The results of this section, in particular integrals, practically do not differ from results, presented in [3].

\subsection{DECAY OF WAVES WITH CIRCULAR POLARIZATION}

Using whirligig principle we expect that in this case one wave with circular polarization will not decay. Decay process will be suppressed.

In this case it is necessary to take into account all components of electromagnetic waves field. Using set of equations (8) - (10) it may obtain following set of shorted equations to define amplitudes $A_{j}^{ \pm}$that slowly varying and describe dynamics wave amplitudes with right and left circular polarization:

$$
\begin{gathered}
\frac{\partial A_{1}^{ \pm}}{\partial t}=-\frac{\omega_{p} \kappa}{2 \omega_{2}} A_{2}^{ \pm} a_{3}, \quad \frac{\partial A_{2}^{ \pm}}{\partial t}=\frac{\omega_{p} \kappa}{2 \omega_{1}} A_{1}^{ \pm} a_{3}^{*}, \\
\frac{\partial a_{3}}{\partial t}=\left(\frac{e}{m}\right)^{2} \frac{\left(k_{1}+k_{2}\right)}{4 \omega_{1} \omega_{2}}\left[A_{1}^{+}\left(A_{2}^{-}\right)^{*}+A_{1}^{-}\left(A_{2}^{+}\right)^{*}\right] .
\end{gathered}
$$

The most essential results that is seen at analysis of third equation of set (15) is fact that amplitude of third wave (low frequency, Langmuir) does not change with time ( $a_{3}=$ const $)$ if field of high frequency wave contains only wave with circular polarization. Really from third equation of set (15) it is seen that multiplier in square brackets of right part of this equation contains 
only products of amplitudes of different polarization. If wave field that decaying contains only one polarization, then factor in square brackets is zero. Thus wave with circular polarization will not take part in three wave interaction (in decay of $t \rightarrow t+l$ type).

\subsection{DECAY OF $t \rightarrow t+l$ TYPE WITH EXTERNAL MAGNETIC FIELD $\omega_{H} \neq 0$}

Let us consider most common case when there are all components of fields of electromagnetic wave and plasma is placed in external magnetic field. The shorted set of equations that describes dynamics of high frequencies transverse wave may be obtained from set (4). Solution for high frequency wave may find in form:

$$
E_{x}=\sum_{j=1}^{2} A_{j}(t) \exp \left(i \varphi_{j}\right)+k . c . ; \quad E_{y}=\sum_{j=1}^{2} B_{j}(t) \exp \left(i \varphi_{j}\right)+k . c .
$$

Then to find dynamics of low frequency waves the following shorted equation is obtained:

$$
\frac{\partial a_{3}}{\partial t}=\left(\frac{e}{m}\right)^{2}\left\{\begin{array}{l}
\left(\frac{k_{1}}{\omega_{1}} A_{1}\right)\left[\frac{i \omega_{2}^{2}}{\left(\omega_{2}^{2}-\omega_{H}^{2}\right)}\left(A_{2}+\frac{\omega_{H}}{i \omega_{2}} B_{2}\right)^{*}\right] \\
+\left(\frac{k_{1}}{\omega_{1}} B_{1}\right) \frac{i \omega_{2}^{2}}{\left(\omega_{2}^{2}-\omega_{H}^{2}\right)}\left(B_{2}-\frac{\omega_{H}}{i \omega_{2}} A_{2}\right)^{*}
\end{array}\right\} .
$$

It is lightly to show that in this case if in the interaction process takes part only one transverse natural wave with circular polarization, then such waves do not decay into transverse and longitudinal ones, i.e. process $t \rightarrow t+l$ can not realize.

To prove this fact enough in formula (17) substitute expression:

$$
E_{x}=\left(E_{+}+E_{-}\right) / 2 ; E_{y}=\left(E_{+}-E_{-}\right) / 2 i \text {. }
$$

Then it is lightly to see when there is only one wave with circular polarization $\left(E_{+}\right.$or $E_{-}$) the right part of equation (15) (and equation (17) too) is zero. This means that such transverse wave which rotates on circle does not excite longitudinal plasma wave and does not decay.

\subsection{INFLUENCE OF FARADEY EFFECT ON DECFY PROCESS}

Above we seen, if in magnetoactive plasma excited only one natural wave, then process of decay will be suppressed. Such process of decay is realized only in that case when in plasma excited two waves with right and left circular polarization. But from whirligig principle it follow, if waves rotate enough fast, this may rip off instability in the magnetoactive plasma wave rotate because of Faraday effect. This is linear process. This rotation can suppress process of decay. It may find condition of such suppression. For this it may find following shorted equations from equations (7) that describe dynamics of waves component at Faraday effect

$$
\dot{E}_{x}=\alpha E_{y} ; \dot{E}_{y}=-\alpha E_{x}
$$

where $\dot{E}=\partial E / \partial t ; \alpha=\omega_{H} \omega_{p}^{2} / 2\left(\omega^{2}-\omega_{H}^{2}\right)<<\omega$.

When formulae were obtained we supposed

$$
\begin{gathered}
\omega_{1} \sim \omega_{2}=\omega ; \omega>>\omega_{H}, \omega_{p} \\
\dot{E}_{ \pm}=\mp i \alpha \cdot E_{ \pm} .
\end{gathered}
$$

Formula (20) point out on fact that natural modes take additional phase dynamics that conditioned by Faraday effect. This additional dynamics will appear as detuning of synchronism, for example, in equation (17), not allowing to excite Langmuir wave. But if amplitude of decaying will be enough large, this detuning will not essentially change dynamics of wave interaction. To define value of this amplitude from equations, for example (15), it is possible to find value of increment of decay instability:

$$
\Gamma \approx(\kappa c) \sqrt{\omega_{p} / \omega} \cdot \varepsilon ; \varepsilon=(e E / m c \omega) .
$$

If value of this increment will be larger than detuning, conditioned by Faraday effect then decay process will be realized. The condition of realization of such decay is inequalities.

$$
\Gamma>\alpha ; \quad \varepsilon>\left(\omega_{H} / \kappa c\right)\left(\omega_{p} / \omega\right)^{3 / 2} .
$$

\section{RESULTS OF NUMERICAL ANALYSIS}

The results described above were obtained by analyzing the shortened equations. Such equations are much simpler than the original equations. However, when they were obtained, many terms and many derivatives were discarded. The question arises: to what extent do the results obtained correctly describe the dynamics of the system under study? To answer this question, it is necessary to find a solution to the original equations. Such a solution can only be obtained by numerical methods. This section presents the results of a numerical analysis of the original equations. Immediately note that the results obtained by numerical methods are in good agreement with the analytical results obtained above.

For comparison the numerical solutions of the original system of equations with the solutions of the shortened system of equations, we will set the initial values of the interacting fields based on the equations of the linear approximation of the original system.

To carry out a numerical analysis of the decay process, it is convenient to introduce dimensionless variables

$$
\begin{aligned}
& \mathrm{a}_{j}=\frac{e E_{j}}{m c \omega_{1}}, \mathrm{a}_{3}=\mathrm{e}_{z}=i \frac{\omega_{p}^{2}}{c \omega_{1} \kappa} \delta n, \beta_{j}=\frac{v_{j}}{c}, \delta n=\frac{\tilde{n}}{n_{0}}, \\
& \tau=\omega_{1} t, k=\frac{\omega_{1}}{c} ; \zeta=k z ; \\
& j=1,2,3-j=1,2 \text { for } H F \text { waves, } j=3 \text { for } L F \text { wave. }
\end{aligned}
$$

For numerical study of the decay dynamics of the linearly polarized wave (Section 1.2), we rewrite the system of equations (14) in the form:

$$
\frac{\partial \mathrm{a}_{1}}{\partial \tau}=-V_{1} \mathrm{a}_{2} \mathrm{a}_{3}, \frac{\partial \mathrm{a}_{2}}{\partial \tau}=V_{2} \mathrm{a}_{1} \mathrm{a}_{3}^{*}, \frac{\partial \mathrm{a}_{3}}{\partial \tau}=V_{3} \mathrm{a}_{1} \mathrm{a}_{2}^{*} .
$$

Here matrix elements $V_{1}=\omega_{p} \kappa c / 2 \omega_{1} \omega_{2}$, $V_{2}=\omega_{p} \kappa c / 2 \omega_{1}^{2}, V_{3}=\left(k_{1}+k_{2}\right) c / 2 \omega_{2}$.

Note that by renormalizing the amplitudes in system (23), the matrix elements $V_{1}, V_{2}, V_{3}$ can always be transformed to the form when they are equal: $V_{1}=V_{2}=V_{3}=V$ (see [3]). For the numerical analysis of the decay process, the following parameters of the system were selected: $f_{1}=2.77 \cdot 10^{9} \mathrm{~s}^{-1}$ is frequency of the HF pump wave, $n_{0}=16 \cdot 10^{9} \mathrm{~cm}^{-3}$ is plasma density, 
$\omega_{2}=\omega_{1}-\omega_{p}$ is frequency of the excited HF-wave, $\omega_{3}=\omega_{p}$ is frequency of the excited LF-wave.

The graphs of the amplitudes dynamics $a_{1}^{2}, a_{2}^{2}, a_{3}^{2}$ versus time are shown in Fig. 3 for stable and unstable (for the case of decay of a linearly polarized wave) for the set values of the wave amplitudes $a 0_{j}=a_{j}(\tau=0)$

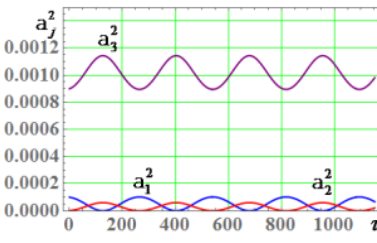

$a$
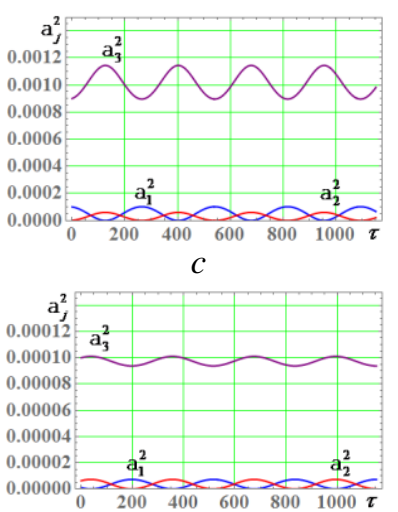

e

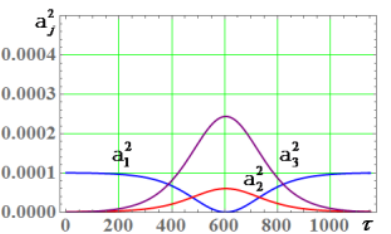

$b$

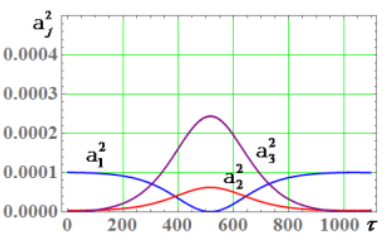

$d$

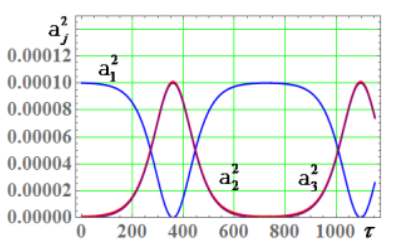

$f$
Fig. 3. Dependences of amplitudes $\mathrm{a}_{1}^{2}, \mathrm{a}_{2}^{2}, \mathrm{a}_{3}^{2}$ of waves with linear polarization on time $\tau: a, b, e, f$-shortened; $c, d$-the original system of equations. Initial values: $\mathrm{a}_{1}=0.01, \mathrm{a}_{2}=0.001(a-d)$;

$$
\mathrm{aO}_{3}=0.03(a, c) ; \mathrm{aO}_{3}=0.0001(b, d) .
$$

For $V 1=V 2=V 3=1: \mathrm{aO}_{1}=0.001, \mathrm{a}_{2}=0.0025$; $\mathrm{aO}_{3}=0.01(e) ; \mathrm{aO}_{1}=0.01, \mathrm{a}_{2}=0.001, \mathrm{a} \mathrm{O}_{3}=0.0001(f)$

As can be seen from the graphs in Fig. 3, the magnitudes of the wave amplitudes are practically the same both in the case of the shortened and the original system of equations. In this case, the small difference in the oscillation period of the amplitudes in the description of the decay by the original and the shortened system of equations is due to the inclusion of cubic and higher order terms in nonlinearity in the original system of equations. At equal values of the matrix elements, the dynamics of the amplitudes of excited waves $\left(a_{2}^{2}, a_{3}^{2}\right)$ practically coincides (see [3]).

The integrals of system (23) (known as the ManleyRowe relations) remain constant (with machine precision) over the entire computation time interval. It follows from these integrals that at the initial amplitudes $V_{2}\left|a_{1}(0)\right|^{2}>V_{1}\left|a_{2}(0)\right|^{2}$ and $V_{3}\left|a_{1}(0)\right|^{2}>V_{1}\left|a_{3}(0)\right|^{2}$ the maximum values of the amplitudes of the decaying waves, depending on the plasma density, are in the relation $\frac{\left|a_{3 \max }\right|^{2}}{\left|a_{2 \max }\right|^{2}}=\frac{\omega_{1}}{\omega_{p}} \frac{\left(k_{1}+k_{2}\right)}{\kappa}$. This dependence is confirmed by the results of numerical analysis.

The obtained results in their physical meaning fully correspond to the results given in [3].
For a numerical analysis of wave decay with circular polarization (Section 1.3), the system of equations (15) is also convenient to rewrite in dimensionless variables $\mathrm{a}_{1,2}^{ \pm}=\frac{e A_{1,2}^{ \pm}}{m c \omega_{1}}, \mathrm{a}_{3}=\frac{a_{3}}{c}$ with the same matrix elements $V_{1}, V_{2}, V_{3}$

Graphs of amplitude dynamics versus time are shown in Fig. 4 for decay of waves with circular polarization when describing this decay by the shortened (26) and the original initial system of equations.
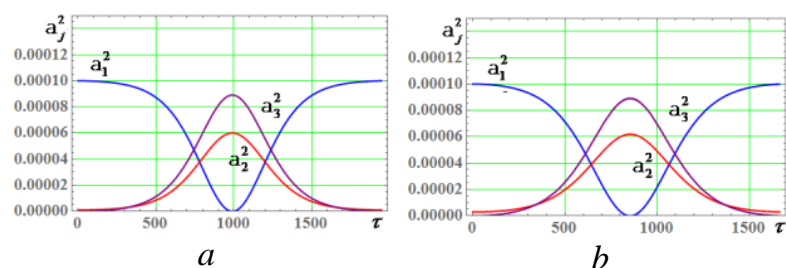

Fig. 4. Dependences of amplitudes $\left(a_{1}^{+}\right)^{2},\left(a_{2}^{+}\right)^{2}, a_{3}^{2}$ of waves with circular polarization on time $\tau$ : Initial

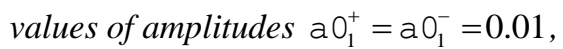

$$
\mathrm{aO}_{2}^{+}=\mathrm{aO}_{2}^{-}=0.001, \mathrm{a} \mathrm{O}_{3}=0.0001 ;
$$

shortened (a); the original system of equations (b)

As can be seen from the graphs in Fig. 4, with circular polarization of waves, the dynamics of the amplitudes of high-frequency waves practically did not change in comparison with the case of waves with linear polarization, but the maximum amplitude of the excited low-frequency wave - the longitudinal velocity $\beta_{z}=a_{3}$ significantly decreased.

If only waves of one circular polarization participate in the decay of the type $t \rightarrow t+l$, then such three-wave interactions of the waves are suppressed.

The results of a numerical study confirm that in the presence of waves of the one circular polarization: $a_{1,2}^{+}(0) \neq 0$ and $a_{1,2}^{-}(0)=0$ or $a_{1,2}^{-}(0) \neq 0$ and $a_{1,2}^{+}(0)=0$ the amplitude of the LF wave remains constant, frequency HF waves is $\omega_{H F}=\left|a_{3}\right| \sqrt{V_{1} V_{2}}$ (Fig. 5).

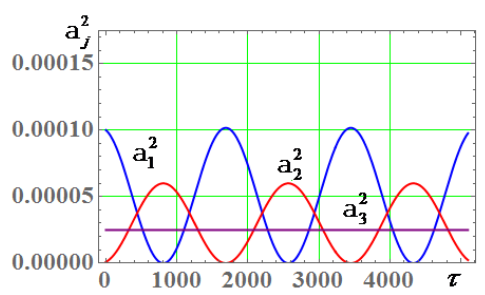

Fig. 5. Dependence of the field amplitude $\mathrm{a}_{3}^{2}$.

On time to time at: $\mathrm{a}_{1}^{+}=0.01, \mathrm{aO}_{2}^{+}=0.001$,

$$
\mathrm{aO}_{1}^{-}=0.0, \mathrm{aO}_{2}^{-}=0.0, \mathrm{aO}_{3}=0.005
$$

The same result takes place in the presence of waves $a_{1}^{-}, a_{2}^{-}, a_{3}^{2}$ and in the absence of waves $a_{1}^{+}, a_{2}^{+}$.

In the presence of an external magnetic field ( $\mathrm{Sec}$ tion 1.4), the dynamics of the LF wave amplitude versus time in the absence of one of the circularly polarized waves completely coincides with the dynamics of the LF wave amplitude in a system without a magnetic field - this amplitude remains constant regardless of the magnitude of the external magnetic field. 
The influence of the detuning caused by the Faraday effect (Section 1.5) on the decay dynamics will be analyzed by numerically solving the original system of equations. The initial values for the fields were chosen to satisfy the linear equations of this system of equations. From the condition for the existence of decay (22), we introduce the threshold value of the pump wave amplitude $\varepsilon_{\text {thres }}\left(H_{0}\right) \approx\left(\omega_{H} / \kappa c\right)\left(\omega_{p} / \omega_{2}\right)^{3 / 2}$, at which the detuning value due to the external magnetic field becomes equal to the increment of the decay process. So with values $H_{0} \approx 40 G \varepsilon_{t h}(40)=0.02$.

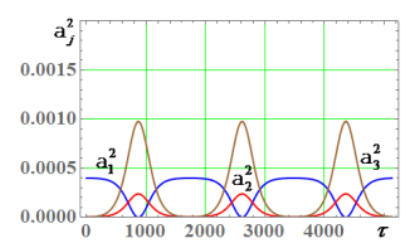

$a$

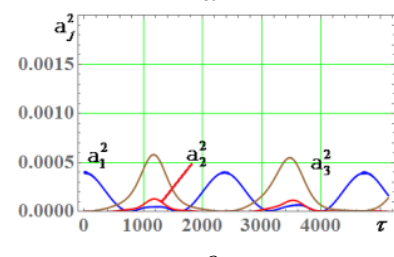

$c$

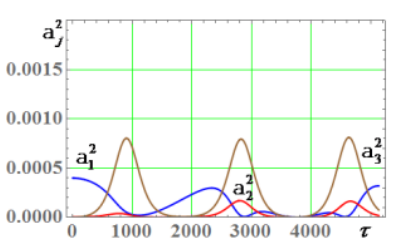

b

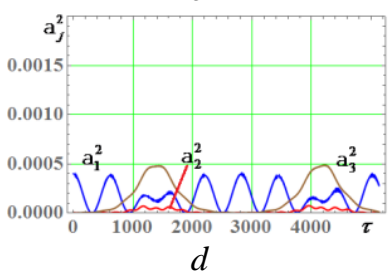

Fig. 6. Dependences of amplitudes $\mathrm{a}_{1 x}^{2}, \mathrm{a}_{2 x}^{2}, \mathrm{a}_{3}^{2}$ on time

for various values of the magnetic field:

$H_{0}=0(a) ; H_{0}=10 G(b) ; H_{0}=25 G(c) ;$

$H_{0}=60 G(d)$. Initial field amplitudes:

$\mathrm{aO}_{1 x}=0.02, \mathrm{aO}_{2 x}=0.001 ; \mathrm{a}_{3}=0.001$

As can be seen from Fig. 6 with an increase in the detuning parameter at $H_{0}=10 G \varepsilon_{\text {thres }}(10) \approx 0.005$, the strict periodicity of the dynamics amplitudes of highfrequency waves was violated, the dynamics of the amplitudes of low-frequency waves remained practically the same as at $H_{0}=0$ with slightly lower maximum values. With an increase in the detuning parameter at $H_{0}=25 G \quad \varepsilon_{\text {thres }}(25) \approx 0.01$, the dynamics of the LF wave amplitude also changes qualitatively, and at $H_{0}=60 G \quad \varepsilon_{\text {thres }}(60) \approx 0.03$ exceeds the initial pump wave amplitude, which leads to the breakdown of the wave decay process.

\section{ACKNOWLEDGEMENTS}

This work was partially supported by the Target Program "Physics of Plasmas and Plasma Electronics: fundamentals and applications" of the National Academy of Sciences of Ukraine (Grant No. 0117U006867).

\section{CONCLUSIONS}

The examples considered above show that the whirligig principle can be used not only to stabilize various instabilities, but also as a tool to predict both the conditions for stable states and the conditions for the development of instabilities. In this work, with his help, it was possible to determine the conditions under which the interactions of waves in a magnetoactive plasma will be unstable, as well as the conditions for the suppression of these instabilities. These results allow us to point out the range of parameters that must be selected to implement the required modes of wave interaction. In particular, it is shown that circularly polarized waves will be stable with respect to decays.

\section{REFERENCES}

1. V.A. Buts. Overview. Mechanisms for increasing the frequency and degree of coherence of radiation // Problems of Theoretical Physics. Series "Problems of Theoretical and Mathematical Physics. Scientific works”. KhGU, 2014, № 1, p. 82-247.

2. V.A. Buts. Quantum Zeno effect, Kapitsa pendulum and Whirligig principle. Comparative analysis // East European Journal of Physics. 2015, v. 2, № 4, p. 4-20.

3. B.B. Kadomtsev. Collective phenomena in plasma. M.: "Nauka" (Gl. Red. Phys.-mat. Lit.). 1988, p. 394 (in Russian).

4. H. Wilhelmsson, J. Weiland. Coherent non-linear interaction of waves in plasmas. M.: "Energoizdat". 1981, 223 p. (in Russian).

5. V.N. Tsitovich. Nonlinear collective effects in plasma. M.: "Nauka", 1967, p. 268 (in Russian).

Article received 16.06.2021

\section{УПРАВЛЕНИЕ ПРОЦЕССАМИ ВЗАИМОДЕЙСТВИЯ ВОЛН В ПЛАЗМЕ}

\section{В.А. Буи, И.К. Ковальчук, А.П. Толстолужсккий, А.Г. Загородний}

Изложены результаты исследования процессов нелинейного взаимодействия волн в плазме. Прежде всего рассмотрены механизмы управления процессами трехчастотного взаимодействия. Показано, что использование принципа юлы позволяет указать на условия существования распадных процессов и на условия подавления процессов распада. Обнаружено, что отдельные волны с круговой поляризацией не распадаются в плазме.

\section{КЕРУВАННЯ ПРОЦЕСАМИ ВЗАСМОДІЇ ХВИЛЬ У ПЛАЗМІ}

\section{В.О. Буц, І.К. Ковальчук, О.П. Толстолужсський, А.Г. Загородній}

Викладені результати дослідження процесів нелінійної взаємодії хвиль у плазмі. Насамперед розглянуті механізми керування процесами тричастотної взаємодії. Показано, що використання принципу дзиги дозволяє вказати на умови існування розпадних процесів і на умови придушення процесів розпаду. Виявлено, що окремі хвилі із круговою поляризацією не розпадаються в плазмі. 